\title{
Wavelet Based Denoising of the Simulated Chest Wall Motion Detected by SFCW Radar
}

\author{
Yunus Emre Acar ${ }^{1}$, Ibrahim Seflek ${ }^{2}$, Ercan Yaldı \\ ${ }^{1}$ Department of Electrical and Electronics Engineering, Faculty of Technology, Selcuk University, Konya, Turkey \\ ${ }^{2}$ Department of Electrical and Electronics Engineering, Faculty of Engineering and Natural Sciences, Konya Technical \\ University, Konya, Turkey \\ *corresponding author, E-mail: yacar@selcuk.edu.tr
}

\begin{abstract}
Low power and compact radars have emerged with the development of electronic technology. This has enabled the use of radars in indoor environments and the realization of many applications. The detection, tracking and classification of human movements by radar are among the remarkable applications. Contactless detection of human vital signs improves the quality of life of patients being kept under observation and facilitates the work of experts. In this study, it was simulated that the movement of the chest wall was modeled and detected by the SFCW radar. Gaussian, Rician and uniformly distributed random noise types were added to the modeled chest motion at different levels. The noisy signal obtained at the receiver is denoised with different mother wavelet functions and the performances of these functions are presented comparatively.
\end{abstract}

\section{Introduction}

With the increasing quality of life, the average life expectancy of individuals is awaited to enhance in the following years. Depending on this, the number of elderly individuals shows parallelism. Therefore, in the future, it will be important to long-term home monitoring the elderly's, the sick and the people in need of care. Traditionally, this process is carried out by connecting probes or sensors to specific parts of the body of individuals. This prevents the individuals to move freely and decreases the quality of life. Both in the context of the comfort of the patients being kept under observation and facilitating the work of the experts, the idea of obtaining non-contact vital signs using radar has been proposed [1-3]. The radars used for military purposes were developed and used for commercial purposes in the 1970s. In the last two decades with the advancing technology, the use of continuous wave radar (CW), ultra-wide band radar (UWB), frequency modulated continuous wave radar (FMCW) and step frequency continuous wave radar (SFCW) has become popular in indoor applications [4]. In addition to the monitoring of the elderly and the patients, burn and newborn cases, obstructive sleep apnea syndrome, security and the through the wall live detection are the areas of application of radars [5-8]. However, performing the applications in the indoor environment causes many unwanted signals that are reflected back from the non-target objects to be perceived as noise in the receiver.

Signals are electrical quantities that provide meaningful information about the behavior of a system. The analysis of any system is directly related to how accurately the signals are processed. One of the most important points of signal processing is to have a noiseless signal. Unfortunately, it is impossible to obtain a noise free signal practically. In this context, one of the most interesting issues about signal processing is to model and eliminate the noise. If a model of the noise can be obtained, it can be eliminated by a reverse operation. There are several noise sources when real world problems are studied. Therefore, it is very difficult to model accurately the noise affecting a system. On the other hand, the distribution of the noise on a signal can be analyzed. It has been shown that different methods are successful to eliminate the various types of noise in many studies [9-13]. In noise removal operations, it is searched for a space in which the signal and the noise can be easily separated. After determining the space, the noisy signal is projected onto a line and the signal is denoised by a simple thresholding. In general the signals are moved from the time domain to the frequency or scale domains for the thresholding process. In order to change the domain Fourier Transform (FT), Short Time Fourier Transform (STFT) and Wavelet Transform (WT) is often used in literature. While FT permits to get only the frequency content of the signal, STFT and WT provide also time information. Unlike STFT, which provides a uniform time resolution, WT offers various time resolutions. The superiority of WT against conventional methods has been emphasized by many studies.[14-16].

In radar, the noise is caused by receiver circuit elements, detection environment and etc. It can be distributed in various ways and it has great importance to eliminate this noise. Ceylan and Canbilen have removed Random, Gaussian and Rician noises on the images using various Multi Resolution Analysis (MRA) techniques [9].

In this study, firstly, the chest wall motion signal is modeled as the study [17]. The three different types of noise are added to this modeled signal. The received signal obtained by a SFCW radar is denoised by using several mother wavelet functions. The denoising performance of the wavelet functions on the chest wall motion signal received by the SFCW radar is presented. 


\section{Method}

Unlike traditional continuous wave radars, a SFCW radar system allows to determine the target range. Different from Frequency Modulated Continuous Wave (FMCW) radar, it provides high range resolution owing to its higher bandwidth. A chest wall motion model is used as the target of the SFCW radar in this work. The noisy return signal is denoised by using WT with various mother functions. Denoising process is done for different noise distributions. The signal which has been moved to scale-time domain is thresholded. The denoised signal is obtained after inverse WT.

\subsection{Transmitted signal}

In a SFCW radar, the transmitting signal is a continuous wave signal with step frequencies. In a Coherent Processing Interval (CPI), with $n$ frequency steps the transmitting signal $S_{n}(t)$ is expressed as [4]

$$
S_{n}(t)=C_{n}(t) \cos \left(2 \pi\left(f_{0}+n \Delta f\right) t+\theta_{n}\right)
$$

where $\Delta f$ is the amount of the increase in frequency, $\theta_{n}$ is the phase of the step $n, f_{0}$ is the initial frequency and $C_{n}(t)$ is the amplitude value which is decreasing with respect to time. The duration of the each consecutive sinusoid $T_{P}$ is constant and affects the range resolution. The $N$ different frequency steps provide to enhance the range resolution. This is main advantage of the SFCW radar. The higher range resolution is obtained with the higher bandwidth. The range resolution of SFCW radar is evaluated as

$$
\Delta R=\frac{c T_{p}}{2 N}
$$

The time-frequency and time-amplitude form of the transmitting signal is given in Figure 1.

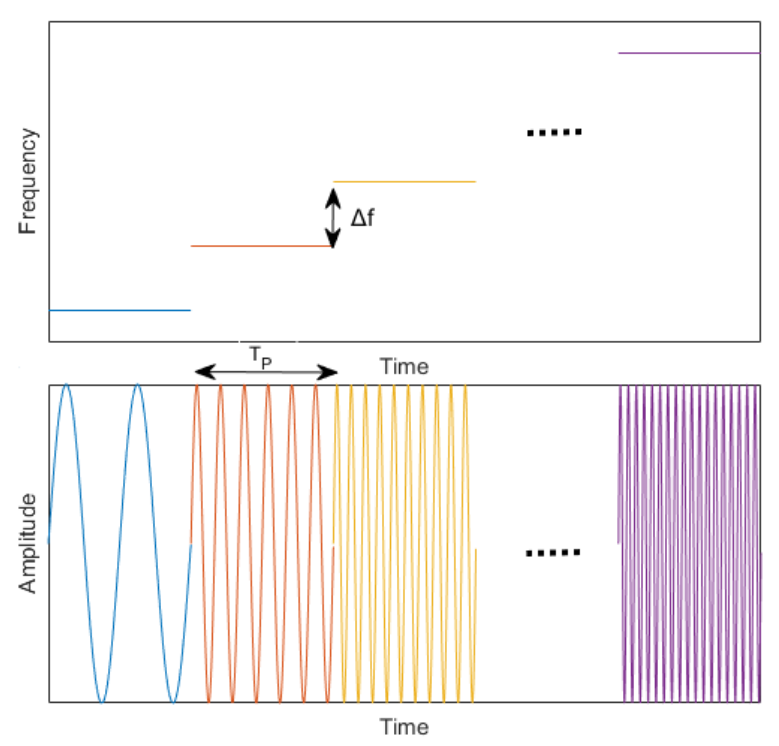

Figure 1: SFCW Radar waveform [4]

\subsection{Received signal}

Doppler theorem is benefitted when SFCW radar is used to detect the vital signs such as respiratory rate and pulse rate. The chest wall displacement of a person is detected first. The respiratory and heartbeat rate can be determined with this information [18-20]. The displacement is calculated using the delay between the signals sent from the transmitter and received from the target. The mathematical expression of the signal reflected from the target is can be written as

$r_{n}(t)=\tilde{C}_{n}(t) \cos \left(2 \pi\left(f_{0}+n \Delta f\right)(t-\tau(t))+\theta_{n}\right)$

where $\tilde{C}_{n}(t)$ is the amplitude value which is decreasing with respect to time and $\tau(t)$ is time delay caused by the chest wall motion. $\tau(t)$ is expressed as [21]

$$
\tau(t)=\frac{R-x(t)}{c / 2}
$$

Where $c$ is the propagation speed of the wave, $R$ is the distance between the radar and the target while $x(t)$ corresponds to the chest wall displacement. When $2 \pi n \Delta f+$ $\theta_{n}$ is an even multiple of $\pi / 2$, null points occur and the displacement can't be evaluated correctly. I/Q modulation/demodulation is one of the popular methods to overcome this problem. When it is used, the transmitted and received signals have both real and imaginary parts. Thus, the received signal is expressed as

$$
r_{n}(t)=\tilde{C}_{n}(t) e^{j\left(2 \pi\left(f_{0}+n \Delta f\right)(t-\tau(t))+\theta_{n}\right)}
$$

After the frequency down conversion and demodulation the receiver signal becomes

$$
y_{n}(t)=\tilde{C}_{n}(t) e^{j 2 \pi n \Delta f \tau(t)}
$$

In order to obtain the displacement $\tau(t)$, FT can be used by considering

$$
\begin{gathered}
y_{n}(t)=\tilde{C}_{n}(t) e^{j 2 \pi F_{s} n T_{p}} \\
F_{s}=\frac{\Delta f \tau(t)}{T_{p}}
\end{gathered}
$$

where $F_{\mathrm{S}}$ is the is the baseband signal frequency.

\subsubsection{Chest wall motion}

There are several ideas to model the chest wall motion in literature. While the displacement signal was taken as a sinusoidal signal in some studies [22], alternatively, in some other works, it was derived from the sinusoidal change of air in the lungs during respiration. A simulated and measured chest wall motion is given in the study of Fauladi and Öncü as in Figure 2 [17]. The movement is divided into 4 separate intervals. The first part of the movement refers to breathing and the second part is a short period of waiting. The third part is exhalation while the last part is relaxation. The mathematical expressions of the signal at the first and third intervals are given in [17] as 


$$
\begin{gathered}
A_{\max } \times \frac{1}{2}\left(1-\cos \left(\frac{\pi t}{T_{1}}\right)\right. \\
A_{\max } \times \frac{1}{2}\left(1-\cos \left(\frac{\pi\left(t-T_{1}-T_{2}\right)}{T_{3}}\right)\right.
\end{gathered}
$$

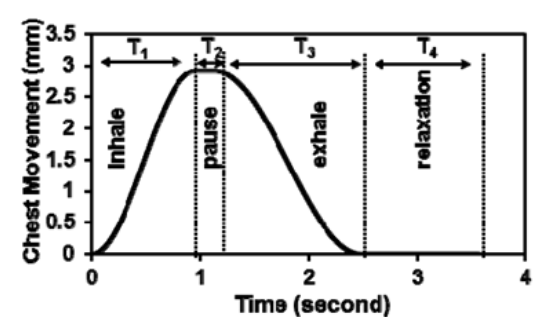

(a)

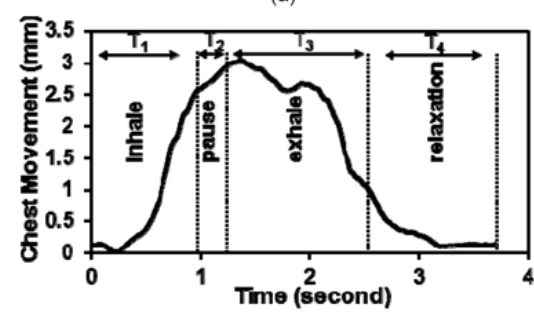

(b)

Figure 2: chest wall motion a) modeled b) measured [17]

\subsubsection{Noise distributions}

In order to process a signal to get the meaningful data, one of the first steps is noise removing. However, the success of the noise removal techniques can alter from one type of the noise to another. This study presents the success of different mother wavelet functions to remove three types of noise. Rician, Gaussian and uniformly distributed random noises are handled. The mathematical expressions of the probability density functions of the noises are given in Equations (11)-(13), respectively. Here, $\mu$ is the mean, $\sigma$ is the standard deviation, $a$ and $b$ are the limits of the random numbers creating the uniformly distributed noise.

$$
\begin{gathered}
f(x \mid \mu, \sigma)=\frac{x}{\sigma^{2}} e^{\left(-\left(\frac{x^{2}+\mu^{2}}{2 \sigma^{2}}\right)\right)} I_{0}\left(\frac{x \mu}{\sigma^{2}}\right) \\
f(x \mid \mu, \sigma)=\frac{1}{\sqrt{2 \pi \sigma^{2}}} e^{\left(-\left(\frac{x-\mu^{2}}{2 \sigma^{2}}\right)\right)} \\
f(x \mid a, b)=\frac{1}{b-a}
\end{gathered}
$$

\subsection{Denoising Process}

In order to remove the noise from the received signal, a suitable domain which is able to separate the noise and the desired signal is searched. While it is not possible to remove the noise in amplitude-time domain, frequency-time or scale-time domains may achieve it. In wavelet based denoising, the amplitude-time domain of the noisy signal is changed to scale-time domain first. Here, noise can be removed by a thresholding process. Then, with the inverse wavelet transform the domain changed back to the original.

\subsubsection{Wavelet transform}

The wavelet transform can be applied discretely and continuously. The discrete and continuous wavelet transforms are defined as

$$
\begin{gathered}
W(j, k)=\sum_{j} \sum_{k} x(k) 2^{-\frac{j}{2}} \psi\left(2^{-j} n-k\right) \\
T(a, b)=\frac{1}{\sqrt{a}} \int_{-\infty}^{\infty} x(t) \psi^{*}\left(\frac{t-b}{a}\right) d t
\end{gathered}
$$

In Equation (14), $\psi(n)$ is called the mother wavelet function while $x(n)$ is the signal to be converted. Similarly, $\psi^{*}(t)$ is conjugate of the mother wavelet function and $x(t)$ is the noisy signal. The parameters $j$ and $a$ are the scale parameters when $k$ and $b$ are the position parameters. With these equations, the wavelet transform can be defined as the sum of the multiplication of the mother wavelet functions and the signal with different scale and positions. Unlike the continuous wavelet transform, discrete wavelet transform calculation is less complex owing to selecting the scaling and position parameters as the power of two. Hence, faster processing capability is achieved in real-time applications.

It is possible to apply the WT repeatedly. In every step, an approximation and a detail signal are created as the output of the WT. After $n$ level transform, we obtain an approximation signal and $n$ detail signals. The sum of these signals gives the main signal. The approximation and detail signals are obtained by low-pass $g[n]$ and high-pass $h[n]$ filtering the main signal. The low frequency approximation signal $y_{a}[k]$ and the high frequency detail signal $y_{y}[k]$ is defined as

$$
\begin{aligned}
& y_{l}[k]=\sum_{n} x[n] g[2 k-n] \\
& y_{h}[k]=\sum_{n} x[n] h[2 k-n]
\end{aligned}
$$

The decomposition of a 3-level discrete wavelet transform is given in Figure 3. As shown in the figure, after filtering, down conversion is done to keep the total signal length constant.

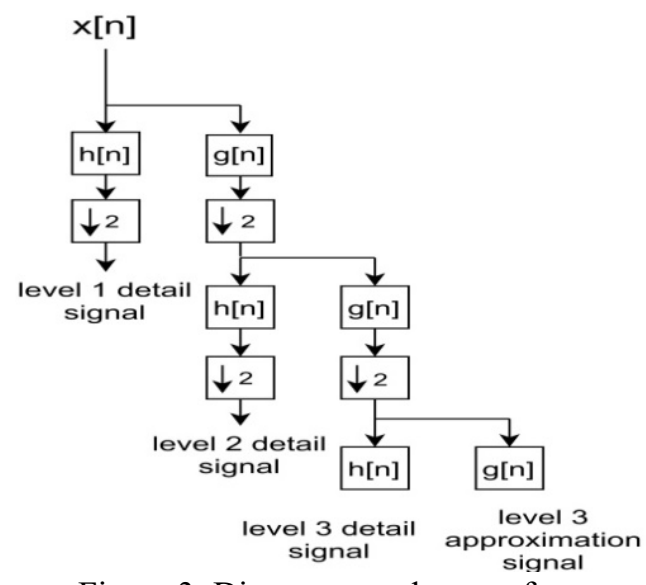

Figure 3: Discrete wavelet transform 


\subsubsection{Thresholding}

Thresholding is the main process in wavelet based denoising. Firstly, a basic threshold level is determined to separate the signal and the noise. This level is calculated depending on the standard deviation of the noise. The standard deviation $(\sigma)$ estimation of the noises are evaluated as in Equation (18). After determining the $\sigma$ the level is calculated as in Equation (19).

$$
\begin{gathered}
\sigma=\frac{\operatorname{median}\left(d_{L-1, k}\right)}{0.6745}, k=0,1, \ldots, 2^{L-1}-1 \\
t=\sigma \sqrt{\log _{2} N}
\end{gathered}
$$

In hard thresholding, the values lower than the thresholding level are forced to be zero while the rest remain unchanged.

$$
\left\{\begin{array}{lll}
y=x & \text { if } & |x| \geq t \\
y=0 & \text { if } & |x|<t
\end{array}\right\}
$$

In soft thresholding, the threshold value is subtracted from the values over the level. The rest are forced to be zero again.

$$
\left\{\begin{array}{lll}
y=\operatorname{sign}(x) \cdot(|x|-t) & \text { if } & |x| \geq t \\
y=0 & \text { if } & |x|<t
\end{array}\right\}
$$

\section{Discussion}

While using SFCW radar, complex samples of the received data whose frequency is down-converted to baseband are placed to a matrix. While the rows of the matrix correspond the consecutive return signals in different frequencies and referred as fast time, the columns correspond the samples of these signals handled as slow time.

Table 1. The I/Q matrix

Sample 1 Sample 2

Sample L

\begin{tabular}{|c|c|c|c|c|}
\cline { 2 - 5 }$f_{0}$ & $\mathrm{I}+\mathrm{JQ}$ & $\mathrm{I}+\mathrm{JQ}$ & $\mathrm{I}+\mathrm{JQ}$ & $\mathrm{I}+\mathrm{JQ}$ \\
\cline { 2 - 5 }$f_{1}$ & $\mathrm{I}+\mathrm{JQ}$ & $\mathrm{I}+\mathrm{JQ}$ & $\mathrm{I}+\mathrm{JQ}$ & $\mathrm{I}+\mathrm{JQ}$ \\
\cline { 2 - 5 } & $\mathrm{I}+\mathrm{JQ}$ & $\mathrm{I}+\mathrm{JQ}$ & $\mathrm{I}+\mathrm{JQ}$ & $\mathrm{I}+\mathrm{JQ}$ \\
\cline { 2 - 5 }$f_{N}$ & $\mathrm{I}+\mathrm{JQ}$ & $\mathrm{I}+\mathrm{JQ}$ & $\mathrm{I}+\mathrm{JQ}$ & $\mathrm{I}+\mathrm{JQ}$ \\
\cline { 2 - 5 } & &
\end{tabular}

In this study, a human chest wall is the target of the SFCW radar. The $x(t)$ signal is defined as mentioned in Equations (9) and (10) with $T_{1}=1, T_{2}=0.25, T_{3}=1.5, T_{4}=1$. The initial frequency is selected as $f_{0}=2 \mathrm{GHz}$ with $\Delta f=$ $20 \mathrm{MHz}$. Each consecutive signal is sampled with $100 \mathrm{~Hz}$. Thus, a $100 \times 375 \mathrm{I} / \mathrm{Q}$ matrix is created. By using the Fourier transform, the range profile is obtained. The modeled and detected chest wall motion is given in Figure 4.
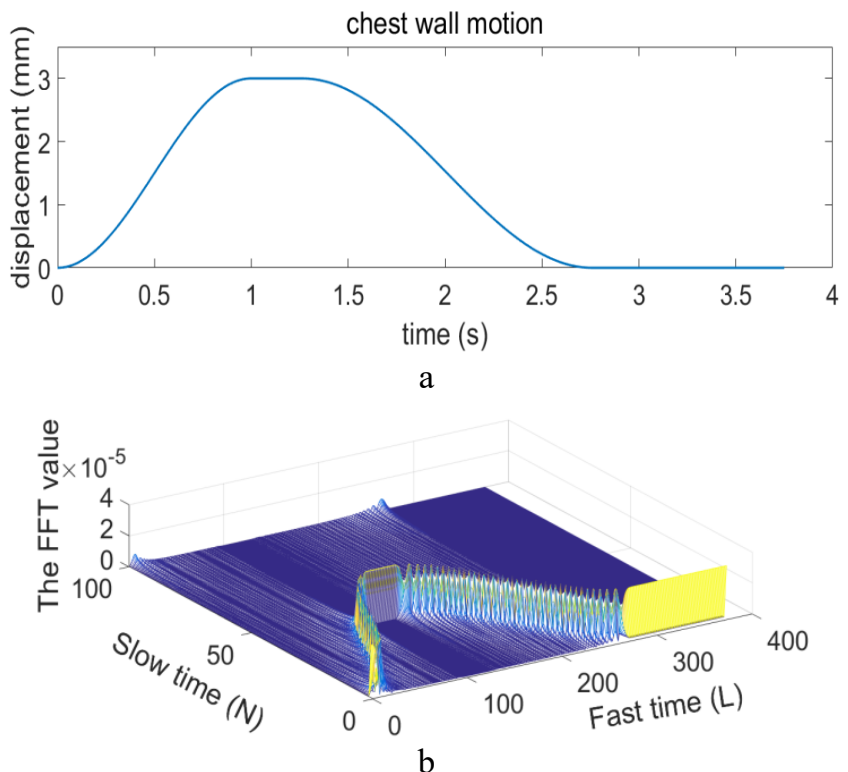

Figure 4: The modeled (a) and received (b) chest wall motion

The range profile evaluation is the step before the WT. After obtaining the range profile, WT, thresholding and the inverse WT steps are carried out and the noise removal operation is finalized. The denoising process of a SFCW radar return signal is given in Figure 5.

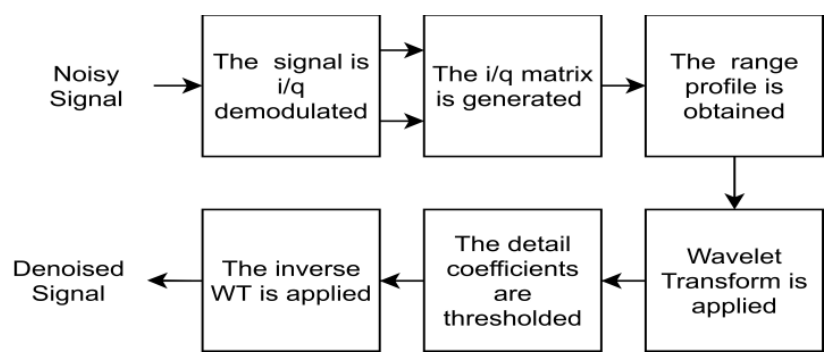

Figure 5: Denoising process of a SFCW radar signal

In order to investigate the power of wavelet transform on different noise levels, the denoising process is done with the SNR of 5, 15 and $25 \mathrm{~dB}$. The Gauss distributed noisy range profiles and their denoised forms are given in Figure 6.
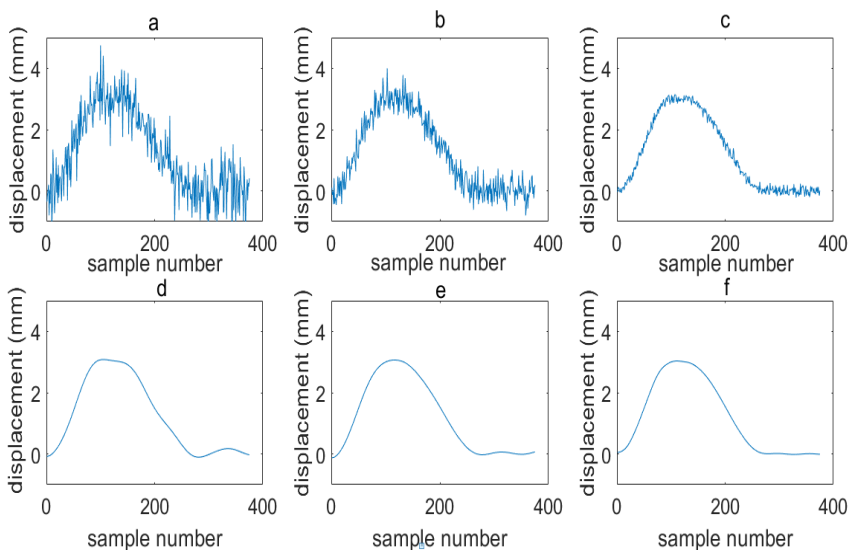

Figure 6: Gauss distributed noisy signals $(\mathrm{a}, \mathrm{b}, \mathrm{c})$ and their denoised forms $(\mathrm{d}, \mathrm{e}, \mathrm{f})$ 

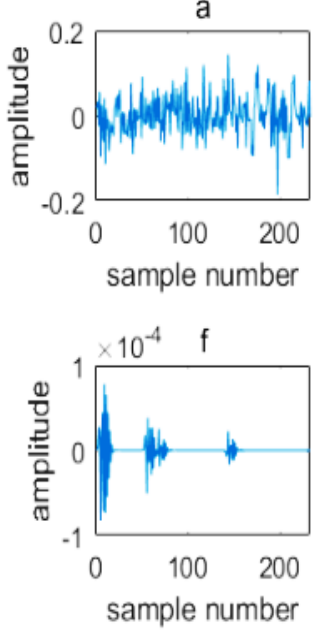

k

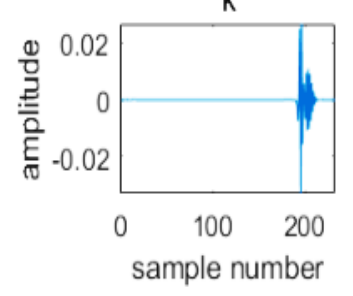

b

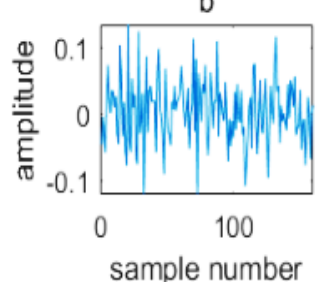

g

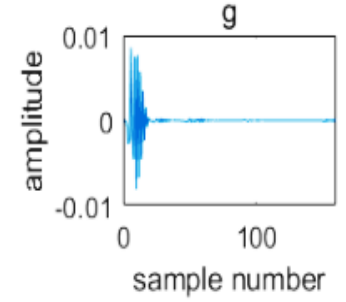

1

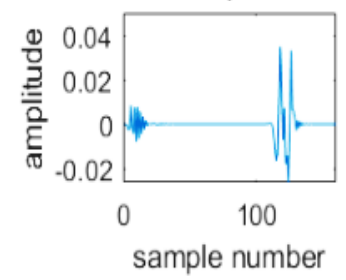

$\mathrm{C}$

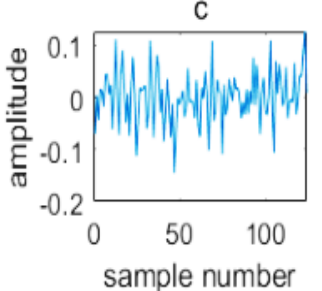

h

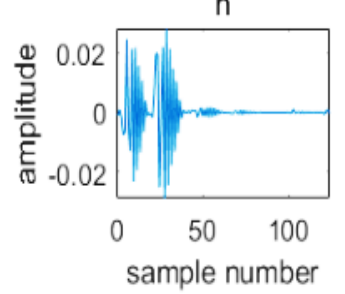

$\mathrm{m}$

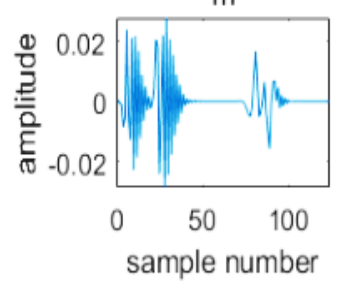

d
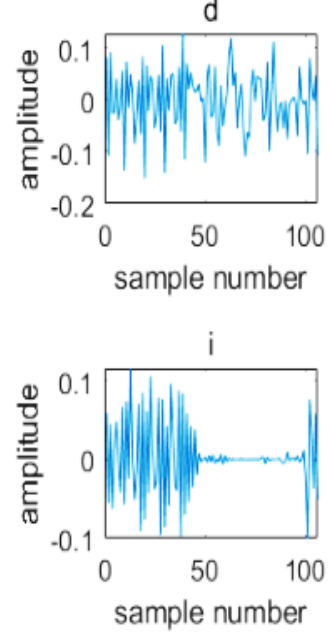

$\mathrm{n}$

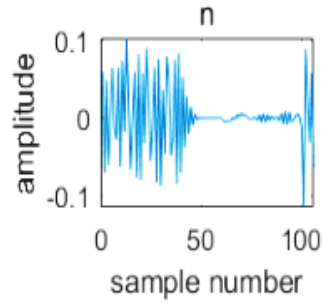

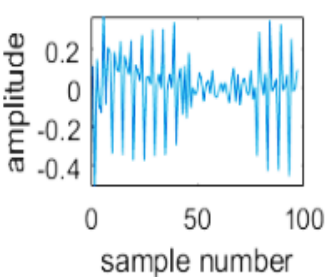

j
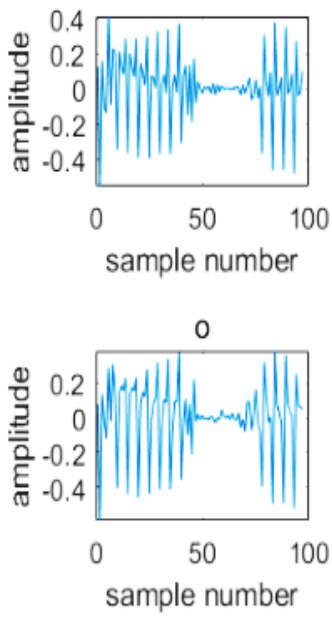

Figure 7. (a-e) 5 level noisy signal details, (f-j) 5 level original signal details and (k-o) 5 level denoised signal details

Daubechies, Coiflets, Symlets, Fejer-Korovkin, Discrete Meyer and Biorthogonal wavelet families, which are popularly used especially in signal processing applications, are used in this study. Each of these families has several members. The difference between the members in a family is the number of vanishing points they used. Considering the one with the minimum number of vanishing points as the simplest, the optimum family member is searched starting from simplest ones. Searching is continued as long as noticeable improvement on SNR is achieved.

The decomposition level is selected as 5 by the experimental results. The thresholding value is estimated with respect to the standard deviation of the first level detail coefficients. Hard thresholding is applied to detail coefficients, while approximation coefficients are remaining unchanged. The original, noisy and denoised detail coefficients for Daub45 are given in Figure 7.

The performance of the wavelet functions are measured with Root Mean Square Error (RMSE) and given in Table 2. As seen from the table, Sym 24 provide the best results for all three SNR levels for the Gauss distributed noise. The Daub45 and Discrete Meyer become stronger when the SNR is higher. None of these functions is clearly superior to remove the Rician distribution, but Discrete Meyer seems the most powerful one. Sym 24 and Bior6.8 are the two functions having least RMSE values for uniformly distributed random noise for these three SNR values.
Table 2: The performance of the Wavelet functions

\begin{tabular}{|c|c|c|c|c|}
\hline SNR & $\begin{array}{l}\text { Wavelet } \\
\text { Function }\end{array}$ & $\begin{array}{c}\text { Norm. } \\
\text { Dist. } \\
\text { Random }\end{array}$ & Gaussian & Rician \\
\hline \multirow{6}{*}{5} & Daub45 & 0,542 & 0,058 & 0,59 \\
\hline & $\begin{array}{l}\text { Discrete } \\
\text { Meyer }\end{array}$ & 0,543 & 0,043 & 0,574 \\
\hline & $F k 22$ & 0,548 & 0,003 & 0,576 \\
\hline & Sym24 & 0,523 & 0,003 & 0,595 \\
\hline & Coif5 & 0,537 & 0,034 & 0,590 \\
\hline & Bior6.8 & 0,542 & $\mathbf{0 , 0 0 3}$ & 0,592 \\
\hline \multirow{6}{*}{10} & Daub45 & 0,250 & 0,019 & 0,314 \\
\hline & $\begin{array}{l}\text { Discrete } \\
\text { Meyer }\end{array}$ & 0,254 & 0,010 & 0,310 \\
\hline & $F k 22$ & 0,263 & 0,010 & 0,327 \\
\hline & Sym 24 & 0,243 & 0,002 & 0,320 \\
\hline & Coif5 & 0,258 & 0,024 & $\mathbf{0 , 3 0 4}$ \\
\hline & Bior6.8 & 0,248 & 0,006 & 0,326 \\
\hline \multirow{6}{*}{20} & Daub45 & 0,091 & 0,001 & 0,095 \\
\hline & $\begin{array}{l}\text { Discrete } \\
\text { Meyer }\end{array}$ & 0,088 & 0,002 & $\mathbf{0 , 0 9 0}$ \\
\hline & $F k 22$ & 0,090 & 0,005 & 0,097 \\
\hline & Sym24 & 0,088 & 0,001 & 0,094 \\
\hline & Coif5 & 0,088 & 0,005 & 0,094 \\
\hline & Bior6. 8 & 0,084 & 0,004 & 0,093 \\
\hline
\end{tabular}

\section{Conclusions}

The contactless measurement of human vital signs has a great importance for many areas. SFCW radars are one of 
the most popular ones in recent years because the fact that it provides a high range resolution. In this study, SFCW radar working principle is handled first. The need of $I / Q$ modulation is underlined. Since the null points cause the range ambiguity, the SFCW radar is considered with $\mathrm{I} / \mathrm{Q}$ modulation/demodulation. Secondly, the usage of the I/Q matrix to obtain the range profile is explained. After mentioning three possible noise distributions briefly, the wavelet transform and thresholding processes are presented. The performance of the six different wavelet functions are compared according to the RMSE they provide. The denoising process is performed for three SNR levels. The results show that the Daub45 and Discrete Meyer wavelet functions becomes more powerful with the increasing SNR for a Gaussian distributed noise when Biort6.8 and Fk22 come to the fore. The results also indicate that the global thresholding with respect to the first level details provide smaller RMSE when Gaussian distributed noise is removed. On the other hand, the method seems weak to handle with the Rician and uniformly distributed noise. Generally, Discrete Meyer does its best for Rician distribution, while Sym 24 is the best choices for uniformly distributed random noise.

\section{References}

[1] M. K. Dremina and L. N. Anishchenko, "Contactless fall detection by means of $\mathrm{CW}$ bioradar," in 2016 Progress in Electromagnetic Research Symposium (PIERS), 2016, pp. 29122915.

[2] G. Sacco, E. Pittella, E. Piuzzi, and S. Pisa, "A radar system for indoor human localization and breath monitoring," in 2018 IEEE International Symposium on Medical Measurements and Applications (MeMeA), 2018, pp. 1-6.

[3] M. Mercuri, P. J. Soh, L. Boccia, D. Schreurs, G. A. Vandenbosch, P. Leroux, et al., "Optimized SFCW radar sensor aiming at fall detection in a real room environment," in Biomedical Wireless Technologies, Networks, and Sensing Systems (BioWireleSS), 2013 IEEE Topical Conference on, 2013, pp. 4-6.

[4] M. Amin, Radar for Indoor Monitoring: Detection, Classification, and Assessment: CRC Press, 2017.

[5] C. Li, J. Cummings, J. Lam, E. Graves, and W. $\mathrm{Wu}$, "Radar remote monitoring of vital signs," IEEE Microwave Magazine, vol. 10, pp. 47-56, 2009.

[6] F. Qi, C. Li, S. Wang, H. Zhang, J. Wang, and G. $\mathrm{Lu}$, "Contact-free detection of obstructive sleep apnea based on wavelet information entropy spectrum using bio-radar," Entropy, vol. 18, p. 306, 2016.

[7] O. Boric-Lubecke, J. Lin, B.-K. Park, C. Li, W. Massagram, V. M. Lubecke, et al., "Battlefield triage life signs detection techniques," in Radar Sensor Technology XII, 2008, p. 69470J.
[8] G. Gennarelli, G. Ludeno, and F. Soldovieri, "Realtime through-wall situation awareness using a microwave Doppler radar sensor," Remote Sensing, vol. 8, p. 621, 2016.

[9] M. Ceylan and A. E. Canbilen, "Performance Comparison of Tetrolet Transform and WaveletBased Transforms for Medical Image Denoising," International Journal of Intelligent Systems and Applications in Engineering, vol. 5, pp. 222-231, 2017.

[10] M. Srivastava, C. L. Anderson, and J. H. Freed, "A New Wavelet Denoising Method for Selecting Decomposition Levels and Noise Thresholds," IEEE Access, vol. 4, pp. 3862-3877, 2016.

[11] R. Hussein, K. BashirShaban, and A. H. El-Hag, "Denoising of acoustic partial discharge signals corrupted with random noise," IEEE Transactions on Dielectrics and Electrical Insulation, vol. 23, pp. 1453-1459, 2016.

[12] R. Mahajan and B. I. Morshed, "Unsupervised Eye Blink Artifact Denoising of EEG Data with Modified Multiscale Sample Entropy, Kurtosis, and Wavelet-ICA," IEEE Journal of Biomedical and Health Informatics, vol. 19, pp. 158-165, 2015.

[13] S. Gaci, "The Use of Wavelet-Based Denoising Techniques to Enhance the First-Arrival Picking on Seismic Traces," IEEE Transactions on Geoscience and Remote Sensing, vol. 52, pp. 4558-4563, 2014.

[14] U. Wiklund, M. Akay, and U. Niklasson, "Shortterm analysis of heart-rate variability of adapted wavelet transforms," IEEE Engineering in Medicine and Biology Magazine, vol. 16, pp. 113118, 1997.

[15] L. Cnockaert, P. Migeotte, L. Daubigny, G. K. Prisk, F. Grenez, S. R. C, et al., "A Method for the Analysis of Respiratory Sinus Arrhythmia Using Continuous Wavelet Transforms," IEEE Transactions on Biomedical Engineering, vol. 55, pp. 1640-1642, 2008.

[16] Z. Dokur, T. Olmez, and E. Yazgan, "Comparison of discrete wavelet and Fourier transforms for ECG beat classification," Electronics Letters, vol. 35, pp. 1502-1504, 1999.

[17] R. F. Fouladi and A. Oncu, "Vital signs modeling for Doppler radar cardiorespiratory monitoring," in Telecommunications and Signal Processing (TSP), 2013 36th International Conference on, 2013, pp. 363-366.

[18] L. Anishchenko, M. Alekhin, A. Tataraidze, S. Ivashov, A. S. Bugaev, and F. Soldovieri, "Application of step-frequency radars in medicine," in Radar Sensor Technology XVIII, 2014, p. 90771N.

[19] G. Gennarelli, F. Soldovieri, L. Marciano, G. Cerasuolo, and O. Petrella, "Measurements performance of a bioradar for human respiration monitoring," Procedia Engineering, vol. 168, pp. 1200-1203, 2016. 
[20] J. Kuutti, M. Paukkunen, M. Aalto, P. Eskelinen, and R. E. Sepponen, "Evaluation of a Doppler radar sensor system for vital signs detection and activity monitoring in a radio-frequency shielded room," Measurement, vol. 68, pp. 135-142, 2015.

[21] D. R. Wehner, "High resolution radar," Norwood, MA, Artech House, Inc., 1987, 484 p., 1987.

[22] J. McNames, J. Bassale, M. Aboy, C. Crespo, and B. Goldstein, "Techniques for the visualization of nonstationary biomedical signals," in Proceedings of the 16th International EURASIP Conference BIOSIGNAL 2002, 2002, pp. 42-45. 